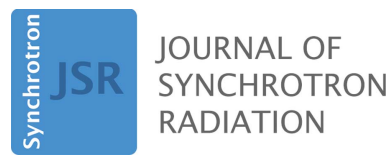

ISSN 1600-5775

Received 26 May 2021

Accepted 15 September 2021

Edited by Y. Amemiya, University of Tokyo, Japan

Keywords: BioSAXS; high flux; ultra-SAXS; microbeam; beamline optics.

Supporting information: this article has supporting information at journals.iucr.org/s

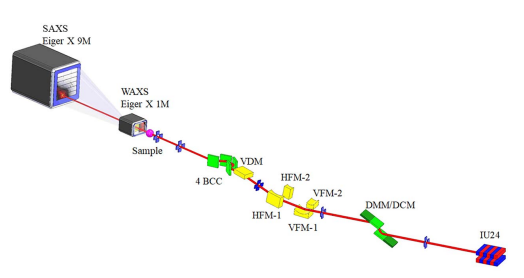

OPEN $\odot$ ACCESS

\section{Optical design and performance of the biological small-angle $X$-ray scattering beamline at the Taiwan Photon Source}

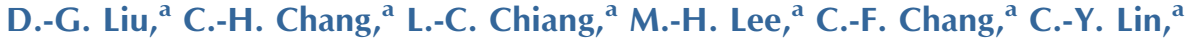 \\ C.-C. Liang, ${ }^{a}$ T.-H. Lee, ${ }^{a}$ S.-W. Lin, ${ }^{a}$ C.-Y. Liu, ${ }^{a}$ C.-S. Hwang, ${ }^{a}$ J.-C. Huang, ${ }^{a}$ \\ C.-K. Kuan, ${ }^{a}$ H.-S. Wang, ${ }^{a}$ Y.-C. Liu, ${ }^{a}$ F.-H. Tseng, ${ }^{a}$ J.-Y. Chuang, ${ }^{a}$ W.-R. Liao, ${ }^{a}$ \\ H.-C. Li, ${ }^{a}$ C.-J. Su, ${ }^{a}$ K.-F. Liao, ${ }^{a}$ Y.-Q. Yeh, ${ }^{a}$ O. Shih, ${ }^{a}$ W.-R. Wu, ${ }^{a}$ C.-A. Wang ${ }^{a}$ and \\ U. Jeng ${ }^{a, b *}$
}

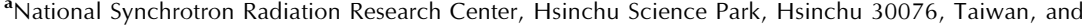
${ }^{\mathbf{b}}$ Department of Chemical Engineering, National Tsing Hua University, Hsinchu 30013, Taiwan.

*Correspondence e-mail: usjeng@nsrrc.org.tw
}

The optical design and performance of the recently opened 13A biological small-angle X-ray scattering (SAXS) beamline at the $3.0 \mathrm{GeV}$ Taiwan Photon Source of the National Synchrotron Radiation Research Center are reported. The beamline is designed for studies of biological structures and kinetics in a wide range of length and time scales, from angstrom to micrometre and from microsecond to minutes. A $4 \mathrm{~m}$ IU24 undulator of the beamline provides highflux X-rays in the energy range $4.0-23.0 \mathrm{keV}$. $\mathrm{MoB}_{4} \mathrm{C}$ double-multilayer and $\mathrm{Si}(111)$ double-crystal monochromators (DMM/DCM) are combined on the same rotating platform for a smooth rotation transition from a high-flux beam of $\sim 4 \times 10^{14}$ photons s$^{-1}$ to a high-energy-resolution beam of $\Delta E / E \simeq 1.5 \times 10^{-4}$; both modes share a constant beam exit. With a set of Kirkpatrick-Baez (KB) mirrors, the X-ray beam is focused to the farthest SAXS detector position, $52 \mathrm{~m}$ from the source. A downstream four-bounce crystal collimator, comprising two sets of $\mathrm{Si}(311)$ double crystals arranged in a dispersive configuration, optionally collimate the DCM (vertically diffracted) beam in the horizontal direction for ultra-SAXS with a minimum scattering vector $q$ down to $0.0004 \AA^{-1}$, which allows resolving ordered $d$-spacing up to $1 \mu \mathrm{m}$. A microbeam, of 10-50 $\mu \mathrm{m}$ beam size, is tailored by a combined set of high-heat-load slits followed by micrometre-precision slits situated at the front-end $15.5 \mathrm{~m}$ position. The second set of $\mathrm{KB}$ mirrors then focus the beam to the $40 \mathrm{~m}$ sample position, with a demagnification ratio of $\sim 1.5$. A detecting system comprising two in-vacuum $\mathrm{X}$-ray pixel detectors is installed to perform synchronized small- and wide-angle $\mathrm{X}$-ray scattering data collections. The observed beamline performance proves the feasibility of having compound features of high flux, microbeam and ultraSAXS in one beamline.

\section{Introduction}

Synchrotron small-angle X-ray scattering (SAXS) beamlines often particularly emphasize on, for instance, biological SAXS (BioSAXS), grazing-incidence SAXS or ultra-SAXS (USAXS), according to the needs of local user communities. Fast user turnover and result publishing are common characteristics of most synchrotron SAXS beamlines. In the past decades, rapid growth in synchrotron flux has made particularly impressive progress on solution SAXS applications. Further facilitated by versatile and mature public SAXS data analysis software packages (Petoukhov et al., 2012), SAXS beamlines for protein solution structures have become increasingly popular and are deployed in many synchrotron 
facilities worldwide (Cowieson et al., 2020; Li et al., 2016; Blanchet et al., 2015).

Previously at the National Synchrotron Radiation Research Center (NSRRC), SAXS activities were initiated in 2002 at the 17B1 wiggler beamline of the synchrotron Taiwan Light Source (TLS) $1.5 \mathrm{GeV}$ storage ring (Hsu et al. 2005). These activities were carried further to the 01B super-bending beamline with an expansion to simultaneous small- and wideangle X-ray scattering (SAXS-WAXS), using two Gabriel gastype linear detectors of length $200 \mathrm{~mm}$ (Lai et al., 2005). After that, a SAXS-WAXS endstation was established at 17B3 beamline in 2006, equipped with a Gabriel-type area detector (200 $\mathrm{mm}$ by $200 \mathrm{~mm}$ ) for SAXS and two linear gas detectors for WAXS (Lai et al., 2006); the gas-type area detector was later upgraded to a MAR165CCD area detector. In 2009, the dedicated NSRRC SAXS beamline 23A was launched (Liu et al., 2009; Jeng et al., 2010) to serve the fast expanding SAXS user community, significantly associated with nanostructural research of block copolymers and nanocomposites. Later, the MAR165CCD detector was replaced by a Pilatus $1 \mathrm{MF}$ area detector of frame rate $133 \mathrm{~Hz}$ (Dectris) for SAXS; the two linear gas detectors were upgraded to two flat-panel detectors CMOS C9827DK and C10158 DK (Hamamatsu) and three Mythen linear detectors of $472 \mathrm{~Hz}$ frame rate (Dectris), packed in a row, for WAXS. In 2016, the $3.0 \mathrm{GeV}$ Taiwan Photon Source (TPS) with high brilliance and low emittance $(1.6 \mathrm{~nm} \mathrm{rad})$ joined operation with the TLS at NSRRC (Horiuchi, 2015), with a steadily increased storage electron beam current from $300 \mathrm{~mA}$ in 2016 to $450 \mathrm{~mA}$ in 2021 . At the port of TPS 13A, an advanced BioSAXS beamline has been jointly developed by NSRRC and Academia Sinica to catch up with the trend research of SAXS on protein solution structures.

The TPS 13A BioSAXS beamline aims to provide high flux and synchronized, time-resolved SAXS-WAXS for studies of biomacromolecular solution structures over a wide range of length and time scales. The beamline also offers USAXS for studies of hierarchical structures of bio-machinery assemblies in solution, gel or condensed forms. Anomalous SAXS/WAXS for metal or mineral distributions and compositions in organelle or drug carriers can also be performed with a wide X-ray energy range covering the calcium $K$-edge. The beamline applications extend to microbeam SAXS/WAXS for correlated crystal and nanostructural mappings in natural fibril tissues and synthetic biomaterials under tailored environmental controls. The optical designs and performance of the new TPS 13A BioSAXS beamline are detailed below.

\section{Result and discussion}

\subsection{Photon source}

The BioSAXS beamline uses the TPS port 13A, having a straight section of $7.0 \mathrm{~m}$ electron beam path, in which space a $4 \mathrm{~m}$ in-vacuum undulator (IU24) (consisting of 168 magnets arranged in a period length of $24 \mathrm{~mm}$ ) was installed (Fig. 1). With the $3.0 \mathrm{GeV}$ electron beam and $500 \mathrm{~mA}$ beam current of
Table 1

Features of IU24 at the minimum gap size of $6.8 \mathrm{~mm}$, under an electron beam of $3 \mathrm{GeV}$ and $500 \mathrm{~mA}$ of TPS.

$\mathrm{H} \times \mathrm{V}$ : horizontal $\times$ vertical directions. $\sigma$ : standard deviation of a normal distribution.

\begin{tabular}{ll}
\hline Parameter & Designed (measured) value \\
\hline Period length & $24 \mathrm{~mm}$ \\
Number of periods & 168 \\
Effective magnetic peak field, $B_{\text {eff }}$ & $0.86 \mathrm{~T}(0.91 \mathrm{~T})$ \\
Effective deflection parameter, $K_{\text {eff }}$ & $1.927(2.03)$ \\
Total magnetic length & $4.032 \mathrm{~m}$ \\
Minimum gap size, $g$ & $6.8 \mathrm{~mm}(6.8 \mathrm{~mm})$ \\
Simulated photon beam size & $120 \mu \mathrm{m} \times 4.3 \mu \mathrm{m} \mathrm{@} 4 \mathrm{keV} ;$ \\
$\quad(\mathrm{H} \times \mathrm{V} ; 1 \sigma)$ & $120 \mu \mathrm{m} \times 2.3 \mu \mathrm{m} \mathrm{@} 24 \mathrm{keV}$ \\
Simulated photon beam divergence & $20 \mu \mathrm{rad} \times 10.1 \mu \mathrm{rad} @ 4 \mathrm{keV} ;$ \\
$(\mathrm{H} \times \mathrm{V} ; 1 \sigma)$ & $19.8 \mu \mathrm{rad} \times 9.7 \mu \mathrm{rad} @ 24 \mathrm{keV}$ \\
\hline
\end{tabular}

TPS, IU24 provides X-rays in the energy range $4.0-23.0 \mathrm{keV}$, covering the $K$-edges of calcium $(4038 \mathrm{eV})$ to ruthenium $(22117 \mathrm{eV})$. The effective magnetic peak field $B_{\text {eff }}$ measured is $0.91 \mathrm{~T}$, with $0.3 \%$ error in field uniformity; a maximum effective deflection parameter $K=2.03$ was derived at $6.8 \mathrm{~mm}$, the designed minimum gap size of IU24. The key parameters of the IU24 are summarized in Table 1. Fig. 1(b) shows the measured and normalized flux spectra of the third and fifth harmonic modes of IU24 at gap 8.0 and $8.7 \mathrm{~mm}$, having peak fluxes at $7376 \mathrm{eV}$ and $4998 \mathrm{eV}$, respectively. The spectra are fitted well using the software SPECTRA (Tanaka \& Kitamura, 2001 ) with input parameters of $3.0 \mathrm{GeV}$ electron beam energy (600 bunches), $30 \mathrm{~mA}$ beam current, and $518.4 \mathrm{~m}$ circumference of the TPS storage ring. The jointly fitted energy spread of $0.11 \%$ of the corresponding $3 \mathrm{GeV}$ electron beam matches closely to the design value of $0.088 \%$.

\subsection{Front-end}

The front-end zone of the beamline outlined in Fig. 2 comprises mainly two X-ray beam-position monitors (XBPM1 and XBPM2) and the high-heat-load slits followed closely by microslits. Each of the XBPMs consists of four CVD diamond blades with multi-metal layer coating for heat dissipation, as detailed previously (Chuang et al., 2020). XBPM1, positioned at $12.35 \mathrm{~m}$, was calibrated via the correlated changes of the diamond blade currents (induced upon beam irradiation) measured over the position scanning of the XBPM in the horizontal and vertical directions, respectively (Chuang et al., 2020). The calibration procedures were further repeated at different IU24 gap sizes (from $7.0 \mathrm{~mm}$ to $10.5 \mathrm{~mm}$, with a step of $0.5 \mathrm{~mm}$ ). The established relationship of the blade-current changes with the XBPM1 positional change (with respect to the beam position) is further parametrized with the changes of the IU24 gap for a compound correlation. The hence calibrated XBPM-1 could report sensitively small beam position changes of a few micrometres during the top-up mode injection of the $3.0 \mathrm{GeV}$ electron beam. Significant positional fluctuations during the IU24 gap change for different X-ray energy could also be observed [Figs. 2(b) and 2(c)]. The watercooled high-heat-load slits comprise two squared frames. The 

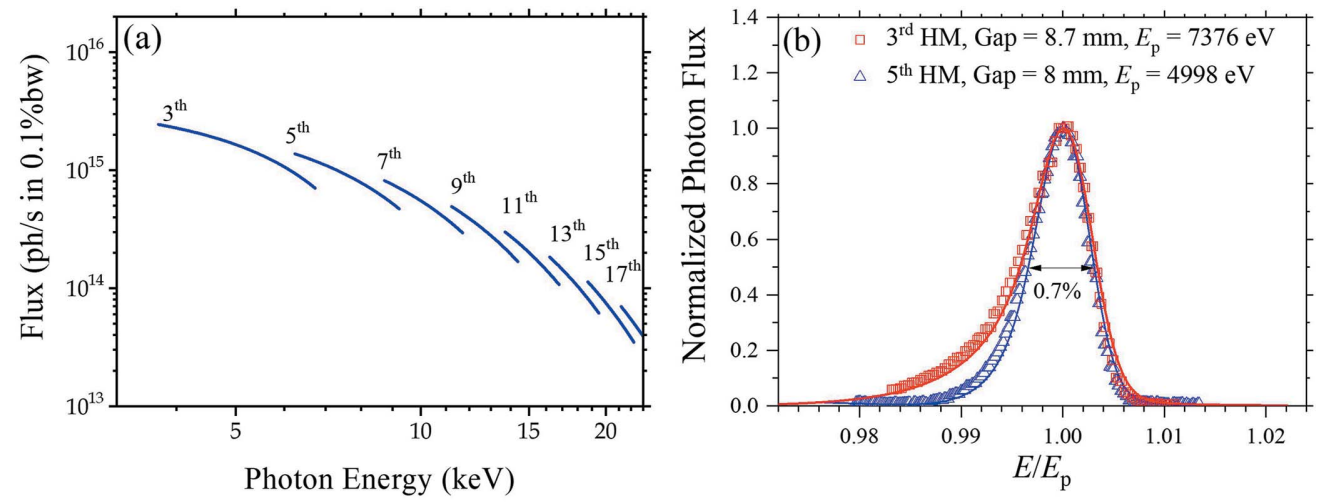

(c)

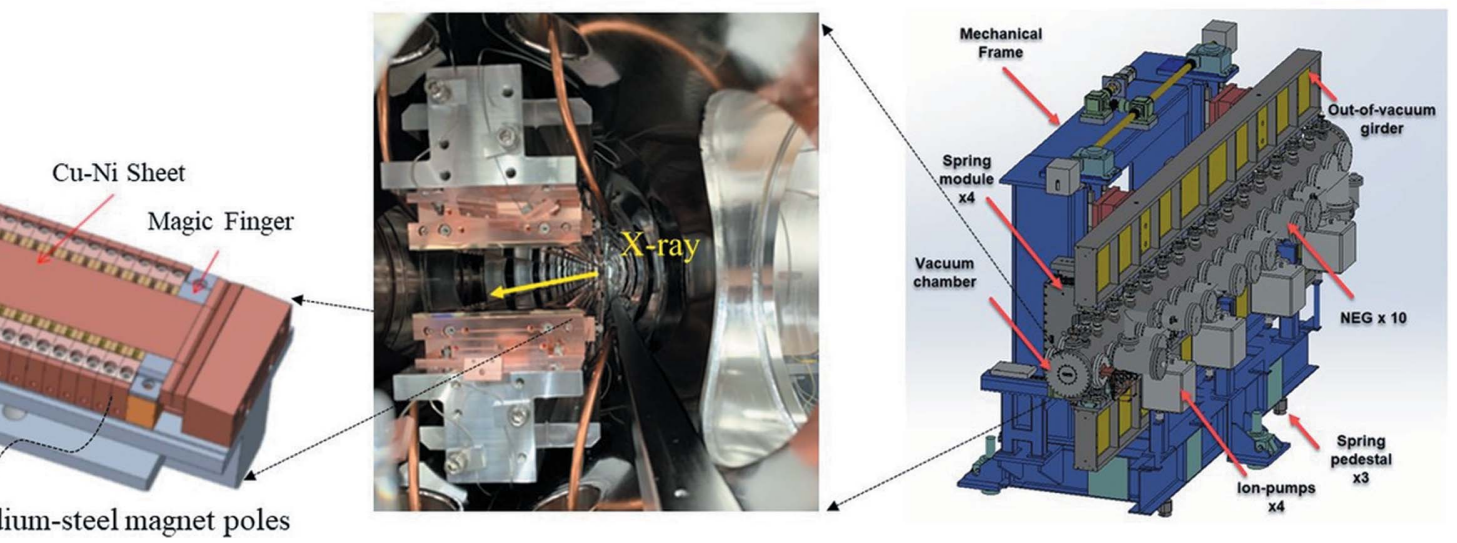

Figure 1

(a) Calculated flux profiles of the harmonic modes (3rd to 17th) of IU24, with the parameters shown in Table 1. (b) Measured and normalized flux spectra of the 3rd and 5th harmonic modes of the undulator IU24 at gaps of 8.0 and $8.7 \mathrm{~mm}$, with flux peaked at $7376 \mathrm{eV}$ and $4998 \mathrm{eV}$, respectively; the data are fitted (solid curves) using the software Spectra. (c) The $4 \mathrm{~m}$ IU24 (right-hand side, with NEG representing for non-evaporable getter pump) comprises up and down vanadium-steel magnet poles (middle) that are sandwiched by blocks of NdFeB alloy. The gap magnet surfaces are covered with $\mathrm{Cu}-\mathrm{Ni}$ sheets (left).

two corners of the squared frames with sharp edges are used to form an aperture down to $50 \mu \mathrm{m}$ size, to reduce the beam size of the strong white beam in microbeam applications; this is followed by a second set of high-precision microslits of four independent tungsten blades to further confine the beam down to $1-50 \mu \mathrm{m}$ [cf. Fig. 2(d)]. Both the high-head-load slits and microslits are framed with invar materials for better thermal stability and positioned on one large piece of granite to reduce environmental vibrations. We note that these microslits can be varied during beamline operation, under an interlock feedback control to prevent the high-precision microslits from over-radiation due to mismoves.

\subsection{Beamline optics}

There are four major operation modes of the new beamline, including (1) the high-flux mode for biomacromolecular solution structures and structural kinetics on a microsecond time scale, (2) USAXS mode for resolving hierarchical structures of bio-machinery assemblies of ordered structures up to $\sim 1 \mu \mathrm{m}$, (3) anomalous SAXS (ASAXS) mode for metal or mineral distributions in bio-structures such as liposome drug-carriers, and (4) microbeam SAXS/WAXS for structural mapping of natural/synthetic tissues or textures. The corresponding beamline optics are designed to cover the needs with easy operations of the four modes (Fig. 3). The major optical components include integrated double-crystal/multilayer monochromators (DMM/DCM) for alternative high-energyresolution mode $\left(\Delta E / E=1.5 \times 10^{-4}\right)$ and high-flux mode $\left(\Delta E / E=8.0 \times 10^{-3}\right)$. The optical system is continued with two parallel sets of beam focusing systems; each comprises a vertical focusing mirror (VFM) facing upward and a horizontal focusing mirror (HFM) facing inward (relative to the storage ring). The focused beam is then leveled by a vertical deflection mirror (VDM), and selectively collimated in the horizontal direction by a downstream four-bounce crystal collimator (4BCC) for USAXS. The corresponding optical systems are detailed further below. We emphasize the largely overlapped beam paths of these characteristic operation modes that allow easy conversions among the operation modes via moving the relevant optical components in and out of the beam path. The corresponding feature parameters of the operation modes are summarized in Table 2.

2.3.1. DMM/DCM. Following a previous design (Liu et al., 2009), we integrate $\mathrm{Mo} / \mathrm{B}_{4} \mathrm{C}$ double-multilayer and $\mathrm{Si}(111)$ double-crystal monochromators (DMM/DCM) on one rotating platform [Fig. 4(b)] (see also Table 3) to provide a fast conversion between the high-flux DMM mode $\left(\sim 10^{14}\right.$ photons s $^{-1}$ in $\left.7-15 \mathrm{keV}\right)$ and the high-energy-resolution $\left(\Delta E / E \simeq 2 \times 10^{-4}\right)$ DCM mode (4-23 keV). The DMM 
(a)

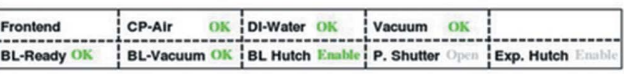

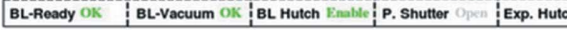
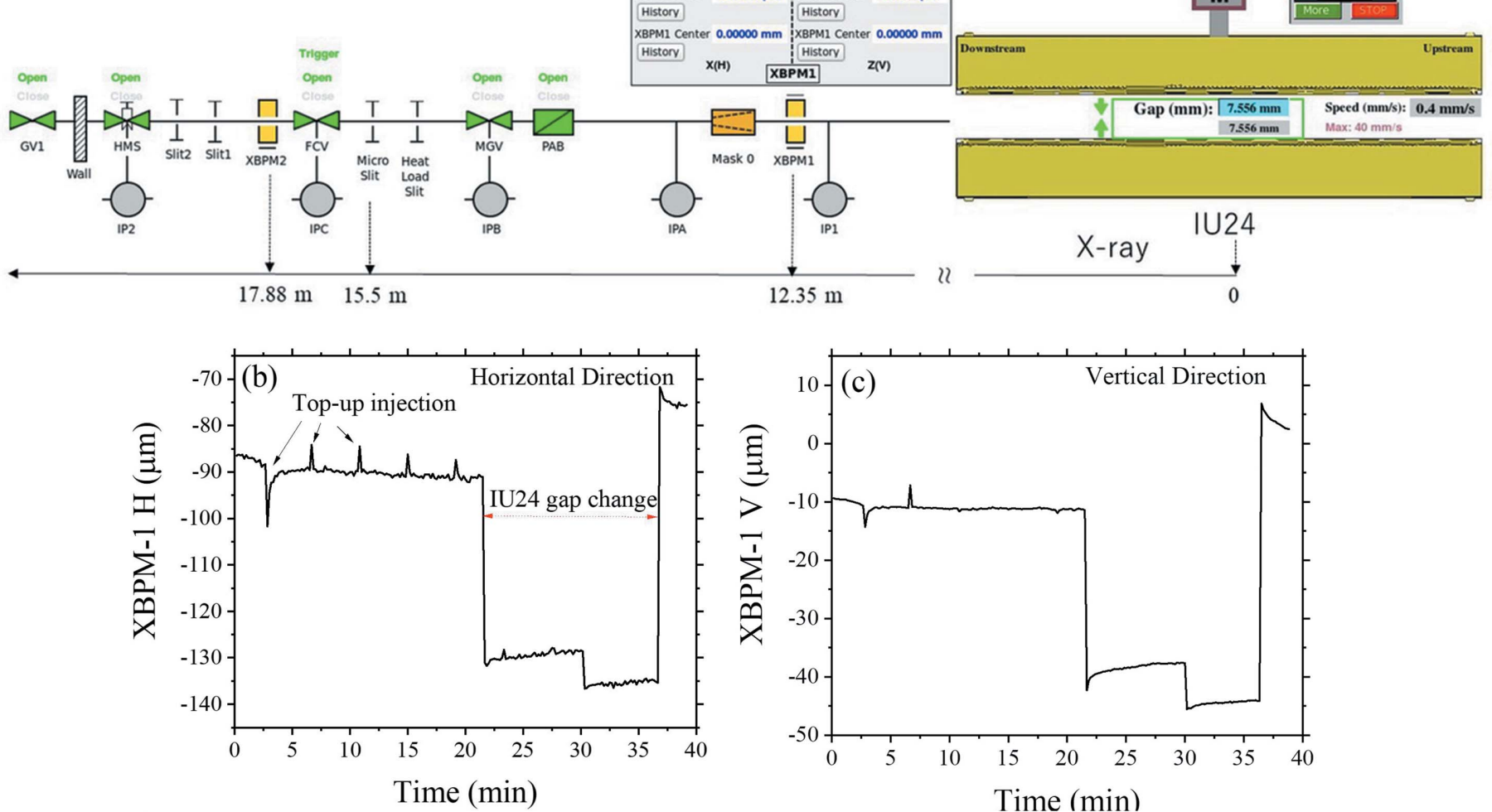

(d)

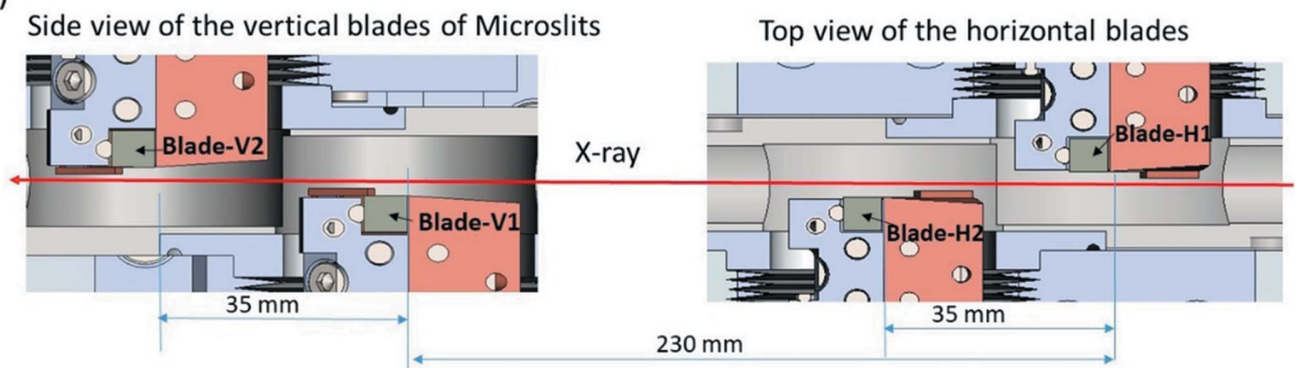

Figure 2

(a) Front-end layout of the TPS 13A beamline, featured with the X-ray beam position monitors 1 and 2 (XBPM1 and XBPM2), high-heat-load slits, and microslits. Panels $(b)$ and $(c)$ are typical beam positions traced in the horizontal and vertical directions by XBPM-1 with $1 \mu \mathrm{m}$ resolution. Periodic beamposition fluctuations of 5-30 $\mu \mathrm{m}$ correspond to the TPS top-up injections of period $4 \mathrm{~min}$. Also shown in the same time interval is a relatively large beamposition shifting during an occasional change of the IU24 gap. (d) Schematics of the microslits, comprising four independent tungsten blades, H1, H2, V1 and V2, separated closely along the beam path for better heat dissipation through individual contacts with copper blocks (colored in orange). The two water-cooled slits of slit1 and slit2 after XBPM2 are optionally used to reduce stray irradiation, hence heat-load on the downstream DCM/DMM. The abbreviations used in (a) are PAB (photon absorber), MGV (metal gate valve), FCV (fast closing valve), DI water (deionized water), CP-Air (compressed air pressure), XBPM (X-ray photon beam position monitor), HMS (heavy metal shutter), IPA-C and IP\# (ion pumps) and GV (gate valve).

consists of two sets of multilayers; each has 200 bilayers of $\mathrm{Mo} /$ $\mathrm{B}_{4} \mathrm{C}$ with a measured mean lamellar spacing of $24.5 \AA$ and a root-mean-squared roughness of $1.8 \AA$ [Fig. S2 of the supporting information (SI)]. A cryogenic cooling system (Research Instruments $\mathrm{GmbH}$ ), of $2500 \mathrm{~W}$ cooling capacity with liquid nitrogen $\left(\mathrm{LN}_{2}\right)$, is used to dissipate a maximum heat-load of $31.4 \mathrm{~W}$ from the DCM crystals or the DMM multilayers after the TPS $13 \mathrm{~A}$ impinging X-ray beam. The designed cooling power could keep the surfaces of the multilayers at $85 \mathrm{~K}$ or the $\mathrm{Si}(111)$ crystal surfaces at $102 \mathrm{~K}$, for which the cooling performance could keep the corresponding slope errors (at the beam spots) below $\sim 1.5$ and $4 \mu \mathrm{rad}$ (calculated values), respectively. $\mathrm{LN}_{2}$ is supplied to the beamline from a central-regulated circulating system of NSRRC. Two diamond filters (100 and $200 \mu \mathrm{m}$ thick) are placed before the DMM/DCM, to selectively attenuate lowenergy X-rays for reduced slope errors of the DCM crystals especially when subjected to $\mathrm{X}$-rays with high-angle incidence.

The flux spectra measured at the $40 \mathrm{~m}$ sample position with the DMM/DCM mode (without 4BCC) match largely with the calculated fluxes [Fig. 4(a)], despite slightly larger deviations observed in the region of higher X-ray energy. We note that 
Table 2

Designed features of the four operational modes of TPS 13A, with beam sizes and divergences in full width at half-maximum (FWHM, $2.35 \sigma$ ). Note that the IU24 source sizes and divergences shown in Table 1 are with $1 \sigma$. Microbeam features are calculated with a microslits opening of $10 \mu \mathrm{m}$ by $10 \mu \mathrm{m}$.

\begin{tabular}{|c|c|c|c|c|}
\hline Operational modes & High flux (DMM) & USAXS Si(111) / Si(311) & ASAXS (DCM) & Microbeam (DCM) \\
\hline Operation energy range $(\mathrm{keV})$ & $7-15$ & $4-15$ & $4-23$ & $4-23$ \\
\hline Horizontal demagnification & & $1.36: 1$ & & $1.61: 1$ \\
\hline Vertical demagnification & & $1.43: 1$ & & $1.45: 1$ \\
\hline Beam divergence $(\mu \mathrm{rad})$ & $60 \times 29$ & $32 \times 29 / 12 \times 29$ & $60 \times 29$ & $70 \times 3.8$ \\
\hline Energy resolution & $0.8 \%$ & & $0.02 \%$ & \\
\hline Flux (photons s ${ }^{-1}$ ) @ $500 \mathrm{~mA}$ & $4 \times 10^{14}$ & $\sim 10^{12} / \sim 10^{11}$ & $2 \times 10^{13}$ & $1 \times 10^{10}$ \\
\hline
\end{tabular}

the flux spectra were measured from the sum current of the quadrant-diamond XBPM Civi-2 (situated at the $40 \mathrm{~m}$ sample position), following a previous report (Desjardins et al., 2018). In the flux deduction, all absorptions from the filters and windows along the beam path were taken into account, including the attenuation due to the two diamond filters and the thin metal coatings of Rigi and Civi-2 XBPMs, and the Be windows. The observed deviations in the measured and calculated flux spectra might associate with the higher transmission (hence lower detecting efficiency) of the quadrant diamond XBPM Civi-2 to high-energy X-rays. Further, with $\Delta E / E=0.8 \%$ close to the energy width of $0.7 \%$ of the X-ray harmonic modes of IU24 [Fig. 1(b)], the DMM provides monochromatic beams in the energy range $7-15 \mathrm{keV}$, with beam intensity 20-30 times higher than that of the corre-

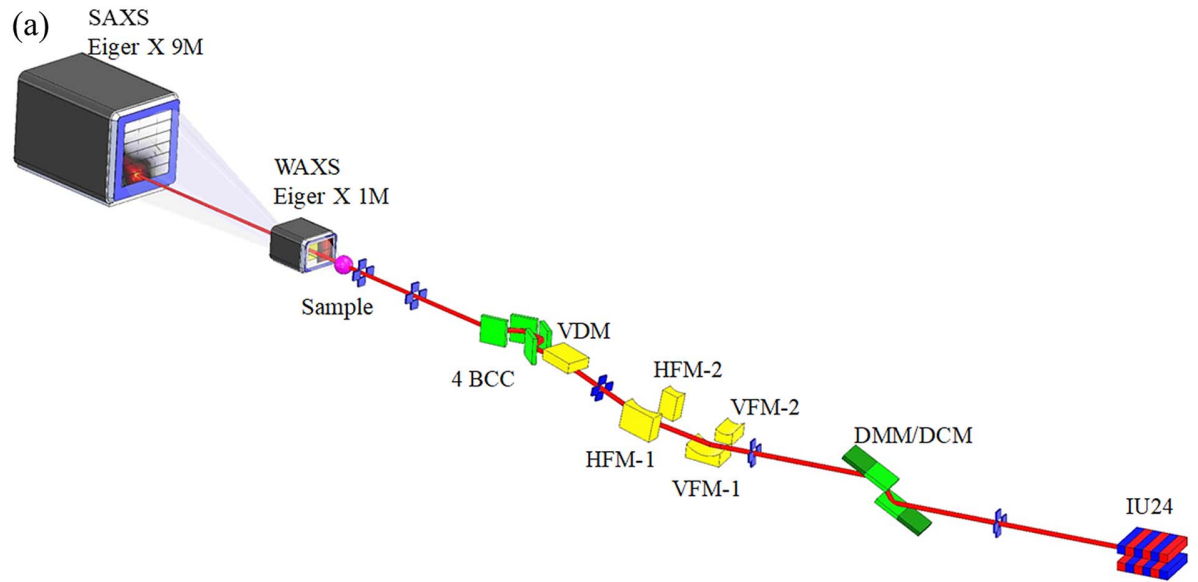

$\longleftrightarrow$ Endstation $(36.7-54.8 \mathrm{~m}) \longrightarrow \leftrightarrow$ Optical Zone $(21.2-36.7 \mathrm{~m}) \longrightarrow \stackrel{\text { Frontend }}{\longleftrightarrow}$

(b)

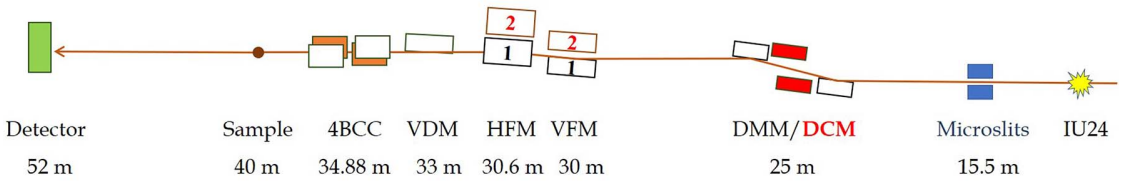

Figure 3

(a) 3D drawing and (b) side view of the primary optical components and beam path of the TPS 13A BioSAXS beamline. Starting from the right are the undulator IU24, microslits, DMM/DCM, two parallel sets of vertical/horizontal KB focusing mirrors (VFM-1/-2 and HFM-1/-2), vertical deflecting mirror (VDM) and four-bounce double-crystal collimator (4BCC). Located in the endstation zone are the sample stage and the detecting system comprising Eiger X 1M for WAXS and Eiger X 9M for SAXS data collections. sponding DCM beam. We note that the energy-dependent $\mathrm{X}$-ray reflectivity of each set of the DMM multilayers (from $58 \%$ of $7 \mathrm{keV}$ to $85 \%$ of $15 \mathrm{keV}$ ) (Fig. S2, SI) affects critically the DMM performance.

The energy resolution of the DCM beam was calibrated with the absorption spectra of ten standard foils that were selectively moved into the beam path (not far from the DMM/ DCM). Shown in Fig. 4(c) is a typical absorption spectrum of the beamline Ti foil, revealing an energy resolution of $\Delta E / E \simeq$ $2 \times 10^{-4}$ of the DCM beam. After a systematic energy offset corrected on the basis of the absorption spectra of the ten foils, the DCM could precisely select the beam energy with an cy of a couple of eV, as shown in Fig. 4(d).

2.3.2. Twin focusing system. Fig. 5(a) shows a twin focusing system containing two sets of Kirkpatrick-Baez (KB) focusing mirrors (located side by side for easy swapping) to selectively focus the beam (with $3 \mathrm{mrad}$ incidence) either to the farthest detector position (detectorfocusing) for general SAXS or to the sample position for microbeam SAXS (sample-focusing). These mirrors are made of silicon crystal blocks (manufactured by JTEC Co.) of $\sim 0.1 \mu \mathrm{rad}$ slope errors and sub-angstrom roughness. Half-strips of the $\mathrm{Si}$ crystal surfaces of the VFM/HFM were coated with a double layer of $25 \mathrm{~nm}$ Pt on top of $5 \mathrm{~nm} \mathrm{Rh}$ for higher X-ray reflectivity with beam energy above $10 \mathrm{keV}$; the other half-strips of bare silicon surfaces of the KB mirrors are used mainly for lower-energy X-rays of $4-10 \mathrm{keV}$, to better suppress the third-harmonic X-rays from IU24. Fig. 5(b) shows that the Si surface has fast decayed reflectivity with X-ray energy above $10 \mathrm{keV}$. High harmonic X-rays (above $12 \mathrm{keV}$ ) can be reduced by a factor below $10^{-3}$ via the three $\mathrm{Si}$-surface reflections from the VFM, HFM and a downstream VDM. The calculated evolutions of beam size and divergence with the detector-focusing optics using a ray- 
Table 3

Length, width and height (L, W and $\mathrm{H}$ ) of the crystals $\mathrm{C} 1$ and $\mathrm{C} 2$ and multi-layers of M1 and M2 of the DMM/DCM shown in Fig. 4(b).

\begin{tabular}{lll}
\hline & C1/M1 & C2/M2 \\
\hline $\operatorname{DCM}(\mathrm{L} \times \mathrm{W} \times \mathrm{H})(\mathrm{mm})$ & $50 \times 40 \times 40$ & $110 \times 50 \times 30$ \\
$\operatorname{DMM}(\mathrm{L} \times \mathrm{W} \times \mathrm{H})(\mathrm{mm})$ & $145 \times 50 \times 30$ & $220 \times 50 \times 30$ \\
\hline
\end{tabular}

tracing program (Sanchez del Rio et al., 2011) are shown in Fig. 5(c). Evolution of the beam size after the sample position was measured with the Eiger X 9M detector (Dectris; pixel size $75 \mu \mathrm{m}$ ), as shown in Fig. 5(d). The result indicates a focused beam size at the designed position at $\sim 52 \mathrm{~m}$ from the source in the vertical direction. Nevertheless, the beam size measured in the horizontal direction shows a minimum at $47 \mathrm{~m}$, suggesting a larger beam incidence on the HFM than the design value of $3.0 \mathrm{mrad}$, leading to a slightly shorter focal length. Fortunately, the beam size is still much smaller than the beamstop ( $4.0 \mathrm{~mm}$ diameter) used, and varies slowly in the detecting range of the SAXS detector; the misfocus of the beam in the high-flux mode operation does not obviously affect SAXS measurements and data quality.

The VFM and HFM of the twin sets of the detector-focusing $\mathrm{KB}$ mirrors and the sample-focusing KB mirrors are situated at $30.0 \mathrm{~m}$ and $30.6 \mathrm{~m}$ positions, respectively, as shown in Fig. $3(b)$. The sample-focusing KB mirrors are designed to focus the beam defined by the microslits (at $15.5 \mathrm{~m}$ position) to the sample $(40 \mathrm{~m})$ position with a small demagnification ratio near 1.5 for microbeam applications. With the microslits opening set to $10 \mu \mathrm{m}$ by $10 \mu \mathrm{m}$ and a $15 \mathrm{keV}$ beam, the microbeam has a minimum vertical beam size of $11.1 \pm 0.8 \mu \mathrm{m}$ (FWHM) at the $40 \mathrm{~m}$ designed focus [Fig. 6(a)] with an optimized flux of $\sim 1 \times 10^{10}$ photons s $^{-1}$ (without the $4 \mathrm{BCC}$ in the beam path); the beam size and flux measured match roughly with the design performance. Nevertheless, the vertical beam size of $9.3 \pm 0.4 \mu \mathrm{m}$ at $37 \mathrm{~m}$ is slightly smaller than that at the design focus, at $40 \mathrm{~m}$, suggesting a slightly off-focused beam in the vertical direction. The horizontal beam dimension measured at the $37 \mathrm{~m}$ position is $23 \mu \mathrm{m}$; however, the beam size measured at the $40 \mathrm{~m}$ focus position is $133 \mu \mathrm{m}$ [Fig. 6(b)]. The misfocus observed might be a result of a significantly larger HFM incident angle used ( $\sim 4.0 \mathrm{mrad})$ compared with the designed $3 \mathrm{mrad}$ incidence; reducing the HFM incident angle toward the designed value however resulted in a significant loss of beam intensity. Improving the horizontal alignment of the central lines of the DCM/DMM and the IU24 source would help resolve the dilemma. We note that such an issue might be circumvented with mirrors of benders for focus tuning, at the expense of the mechanical stability of the mirrors, hence the beam stability.

2.3.3. USAXS with 4 BCC. For USAXS, a four-bounce crystal collimator (4BCC) [Fig. 7(a)] located downstream is used to further horizontally collimate the monochromatic
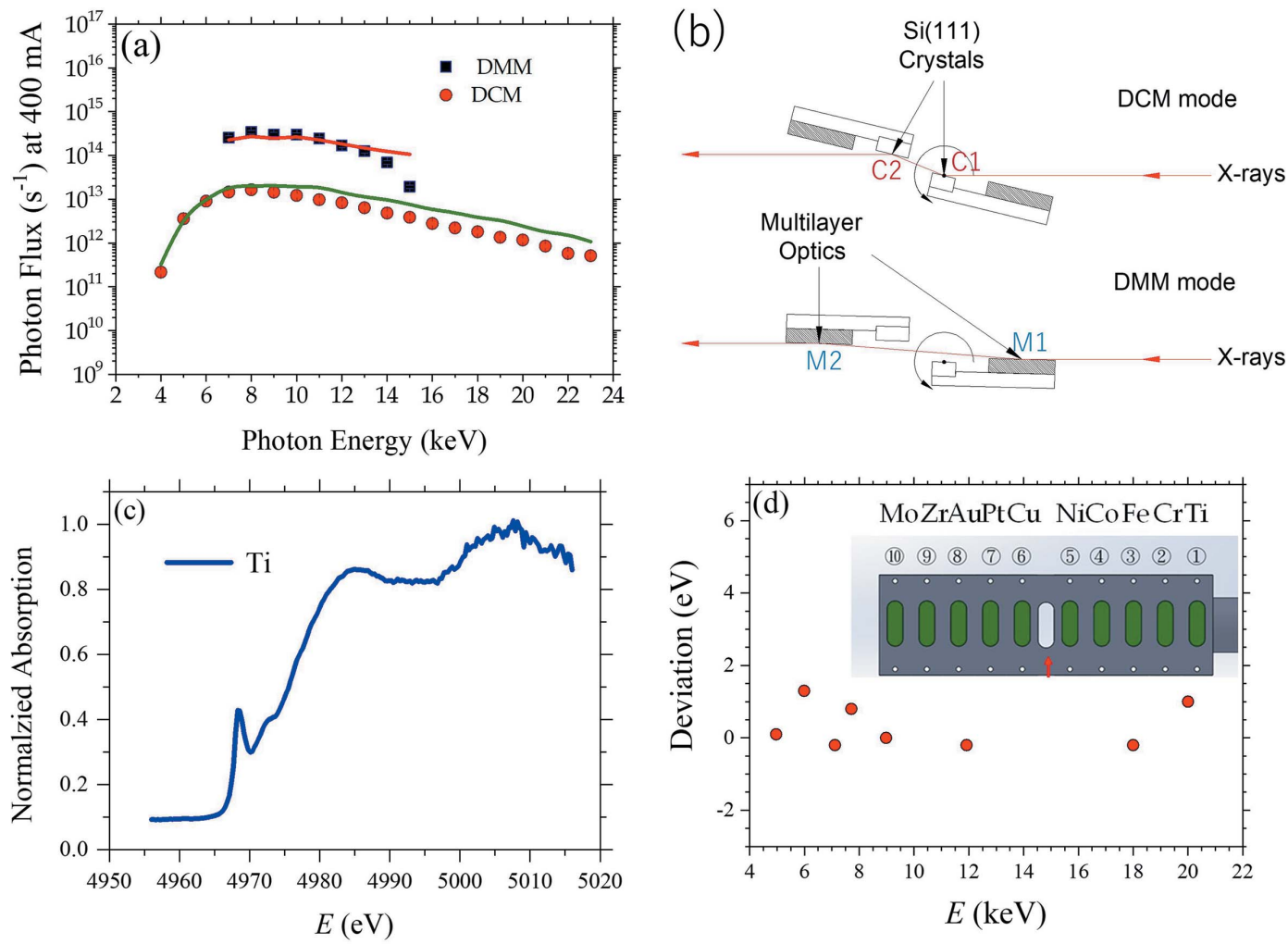

Figure 4

(a) DMM/DCM flux spectra measured at the sample position ( $40 \mathrm{~m}$ from the IU24), with an electron beam current of $400 \mathrm{~mA}$. The spectra are fitted largely well with the calculated spectra (solid curves). (b) Schematic view of the dual monochromators DMM/DCM, with the rotating center of the whole system situated at the center of the first $\mathrm{Si}(111)$ crystal. The rotation range of the $\mathrm{DMM} / \mathrm{DCM}$ platform from $0.965^{\circ}$ to $2.068^{\circ}$ is for a $15-7 \mathrm{keV}$ beam with DMM, and $4.93^{\circ}$ to $29.62^{\circ}$ for $23-4 \mathrm{keV}$ with DCM. (c) A typical absorption spectrum of a standard Ti foil taken with the DCM. $(d)$ Deviation of the DCM beam energy after calibration with the ten in-vacuum standard foils (inset) deployed downstream of the DMM/DCM. 

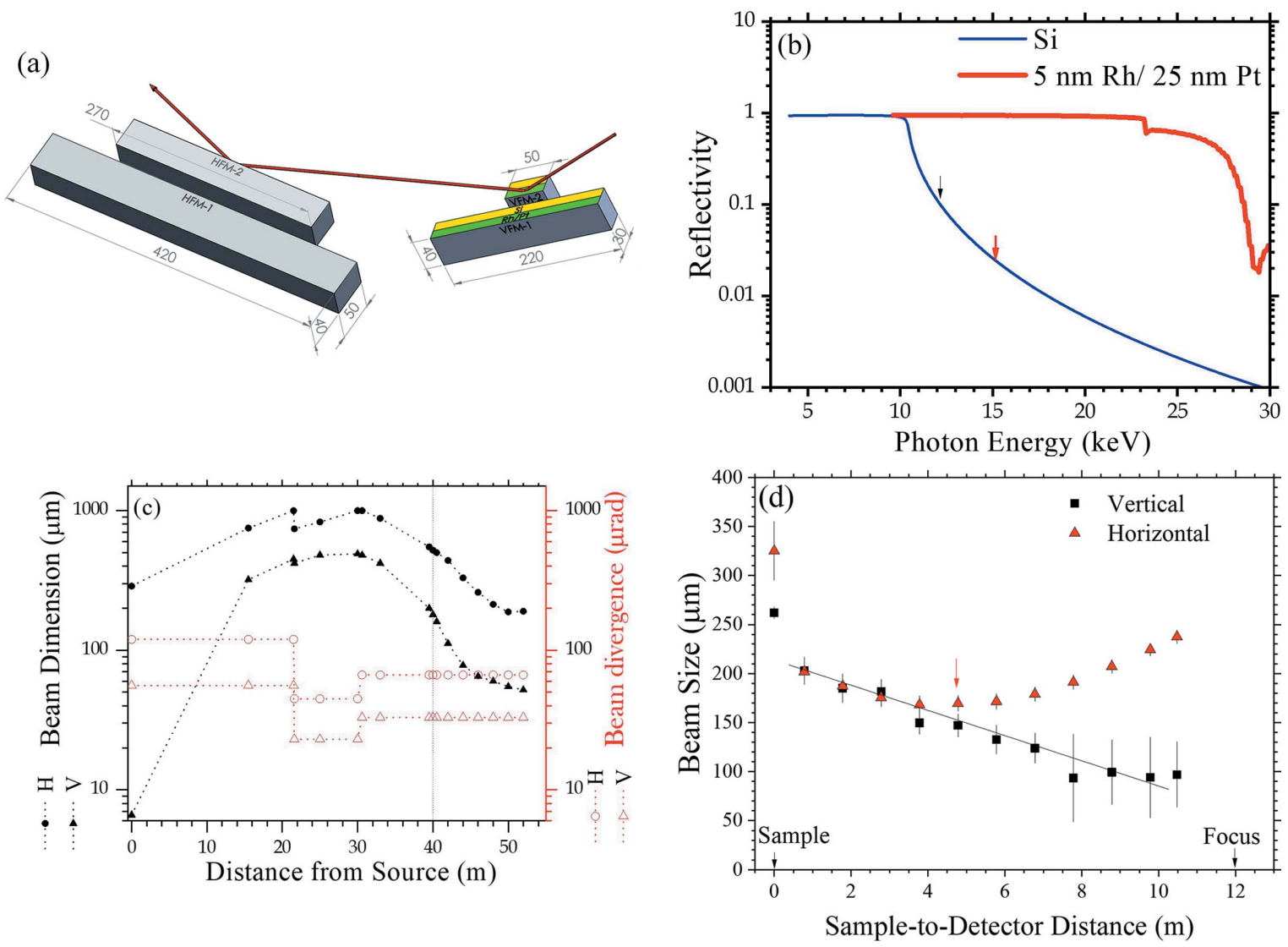

Figure 5

(a) The twin focusing system comprising two sets of vertically and horizontally focusing mirrors (VFM and HFM) made of Si crystal blocks with the indicated dimensions (in $\mathrm{mm}$ ). Half of each mirror surface is coated with $\mathrm{Ru} / \mathrm{Pt}$ bilayers (colored in green) for enhanced reflectivity of high-energy $\mathrm{X}$-rays; the other half remains bare Si surface (yellow). (b) Calculated X-ray reflectivity of the bare Si surface and Pt/Ru-coated surfaces of the focusing mirrors. (c) Simulated beam size and divergence (FWHM) over the whole beam path. (d) Measured evolution of the beam size from the sample position to the beam focus, using a $10 \mathrm{keV} \mathrm{DCM}$ beam and the Eiger X $9 \mathrm{M}$ detector. The beam size $280 \mu \mathrm{m}(\mathrm{H}) \times 50 \mu \mathrm{m}(\mathrm{V})$ extrapolated (solid line) to the focus position is consistent with the simulated one shown in $(c)$. Note that the beam size at the $52 \mathrm{~m}$ focus could not be measured due to a length limit (14 $\mathrm{m}$ ) of the data transmission cables of the Eiger $\mathrm{X} 9 \mathrm{M}$ detector in vacuum.

X-ray beam after the DCM of a vertical diffraction plane. The 4BCC consists of two sets of $\mathrm{Si}(311)$ double-crystal collimators in the horizontal diffraction plane arranged in a dispersive configuration [Fig. 8(b)] to significantly decrease the beam divergence to $\sim 30 \mu \mathrm{rad}$. Initially, $\mathrm{Si}(111)$ crystals were used for establishing the high-demanding alignment procedure of the 4BCC, as detailed in Fig. S1 (SI). Instead of using channelcut crystals of common surface roughness of more than $10 \AA$, the $4 \mathrm{BCC}$ of the beamline adopts four independent superpolished crystals with a surface roughness down to $\sim 2 \AA$ (Sztucki et al., 2019). The rotation axes of the two doublecrystal collimators are located at the upper left corner of the first crystal $\mathrm{C} 1$ and the upper right corner of the fourth crystal $\mathrm{C} 4$. The parallelism of the paired crystals and the relative orientations of the two sets of double-crystal collimators could be fine-tuned to the $1 \mu \mathrm{rad}$ parallelism of the level of the channel-cut crystals, using pico-motors (within $1 \mathrm{mrad}$ range) and piezo actuators $(100 \mu \mathrm{rad}$ range). Moreover, there is a $10 \mathrm{~mm}$-diameter tunnel ( $8 \mathrm{~mm}$ below the crystal surface) in both the $\mathrm{C} 1$ and $\mathrm{C} 4$ crystals, allowing the $\mathrm{X}$-ray beam to bypass the $4 \mathrm{BCC}$ diffraction collimation, when the two sets of double-crystal collimators are rotated to be parallel with the X-ray beam [Fig. 7(b)]. Fig. 7(c) shows that the $4 \mathrm{BCC}$ with
Si(111) (used for a preliminary test) could significantly cut down the $8 \mathrm{keV}$ DCM beam divergence mainly in the horizontal direction for nearly 100 times less background scattering, despite an as-simulated tenfold loss of the X-ray peak intensity to $\sim 10^{11}$ photons s $^{-1}$ (Liu et al., 2019).

2.3.4. Beam diagnostic and control. The quadrant-diamond XBPM-1 (Fig. S3, SI) situated in the front-end zone (before any beamline optical component) is calibrated with different gap-openings of IU24, and can report the beam positions in situ with $1 \mu \mathrm{m}$ resolution. XBPM-1 is also used as a guide in selecting (searching) the local $3 \mathrm{GeV}$ electron beam position and incline angle in IU24, for an optimized beam path to the optical systems of the beamline. Each optical component is equipped with an in-and-out two-position screen made of YAG crystal and a camera readout system for fluorescence beam imaging (50 $\mu \mathrm{m}$ resolution). The beam positions on the screens provide convenient and fast guides in re-alignments and double-checking of the beam path defined by the optical components during conversions of the operation modes or rejections of the $3 \mathrm{GeV}$ electron beam. There are also two FMB-Oxford Nano-XBPMs positioned after the DMM/DCM and $4 \mathrm{BCC}$ for beam imaging with sub-micrometre resolution, whose positional information would be used for potential 

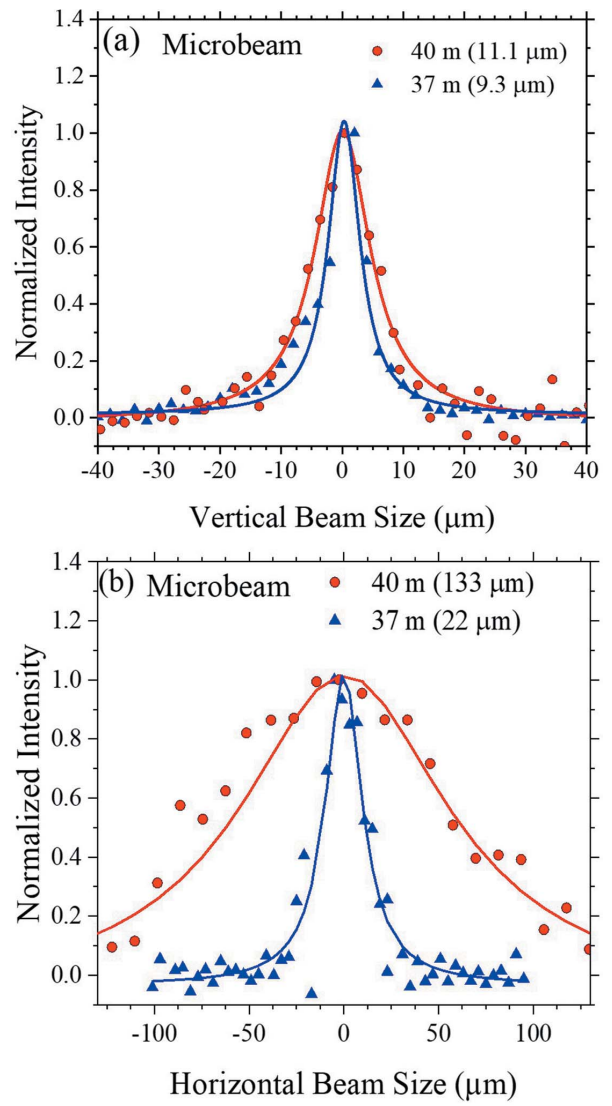

Figure 6

(a) Vertical and $(b)$ horizontal beam profiles of a $15 \mathrm{keV}$ microbeam with DCM and the sample-focusing KB mirrors measured at $37 \mathrm{~m}$ and $40 \mathrm{~m}$ positions, using an opening of $10 \mu \mathrm{m} \times 10 \mu \mathrm{m}$ of the microslits. The fitted beam dimensions (FWHM) are indicated in the brackets.

feedback controls of the optical system for beam-position locking.

The beamline adopts the Experimental Physics and Industrial Control System (EPICS) for integrated controls of the hardware and software (Hatje et al., 2007), including all the motors of the beamline optical components and their corresponding sensing (temperature) and cooling (flow rate) systems. For beamline devices without off-the-shelf EPICS support modules, such as in-house current readout systems of the several quadrant-diamond XBPMs, in-house-made support modules were also collectively implemented into EPICS. Another aspect of the EPICS of the beamline is to serve as a platform in coordinating standalone graphical user interface systems of the beamline add-on devices through the IOC (Input/Output Controller) server program. Communications among local systems are realized via defined process variables (PVs) that can actively propagate out or be accessed timely in the same local networks of the IOC (Chiang et al., 2019). We have integrated two main clients of PVs to the EPICS of the beamline: (1) the graphical user interface of Control-System Studio (CSS) for visual operations, and (2) a UNIX-based software package SPEC (SPEC Control Systems Ltd) for programmable operations of the beamline components and data collection. The CSS provides visual icons, status indicators, interactive dialog inputs for a graphical user
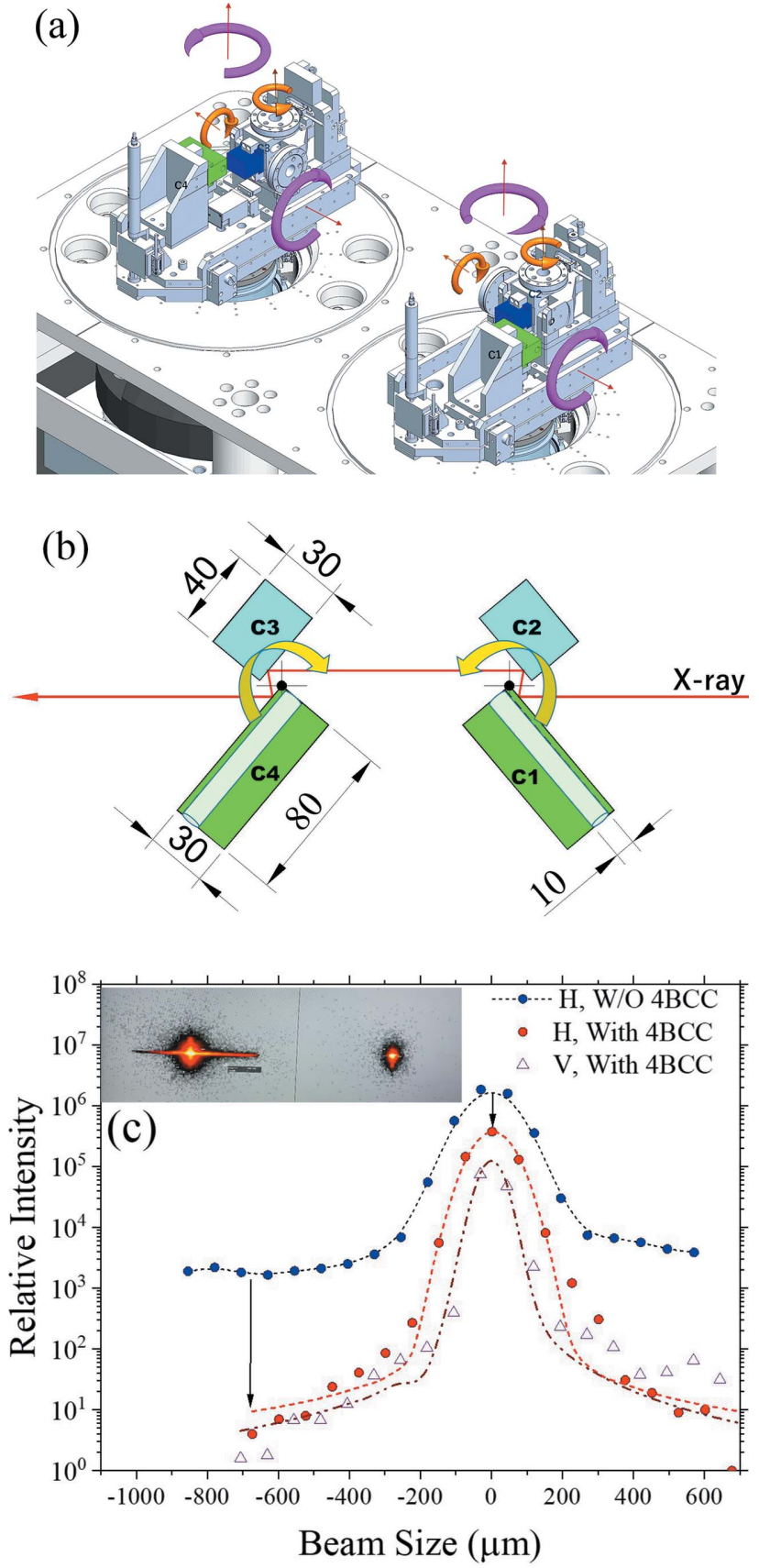

Figure 7

(a) 3D drawing of the four-bounce crystal collimator 4BCC (situated at $34.88 \mathrm{~m}$ position), comprising two sets of double crystals of $\mathrm{Si}(111)$ or $\mathrm{Si}(311)$ situated on two large rotating stages for a dispersive configuration. On each rotation stage, the two crystals $(\mathrm{C} 1-\mathrm{C} 2$ or $\mathrm{C} 3-\mathrm{C} 4)$ are rotated/titled ( $c f$. large arrows) collectively; $\mathrm{C} 2$ and $\mathrm{C} 3$ crystals further can be rotated/titled ( $c f$. small arrows) with respect to the $\mathrm{C} 1$ and $\mathrm{C} 4$ crystals, respectively. A motorized YAG crystal is installed between C3 and $\mathrm{C} 4$ for alignment convenience. The configuration of these two rotation stages can be improved further as detailed in SI. (b) An action mode of the $4 \mathrm{BCC}$ with the photon beam passing through the four crystals of the 4BCC. With 4BCC rotations (indicated by the arrows), $\mathrm{X}$-rays could bypass $4 \mathrm{BCC}$, through the two $80 \mathrm{~mm}$-long channels in crystals $\mathrm{C} 1$ and $\mathrm{C} 4$. (c) Horizontal and vertical beam profiles of a DCM $8 \mathrm{keV}$ beam measured at $49.5 \mathrm{~m}$ near the $52 \mathrm{~m}$ focus, without (2D image to the left, inset) and with (2D image to the right, inset) the 4BCC with $\mathrm{Si}(111)$. Note that the background scattering intensity near the primary beam is decreased by about two orders of magnitude (long arrow) with the $4 \mathrm{BCC}$, at a cost of a tenfold decay in beam central intensity. 


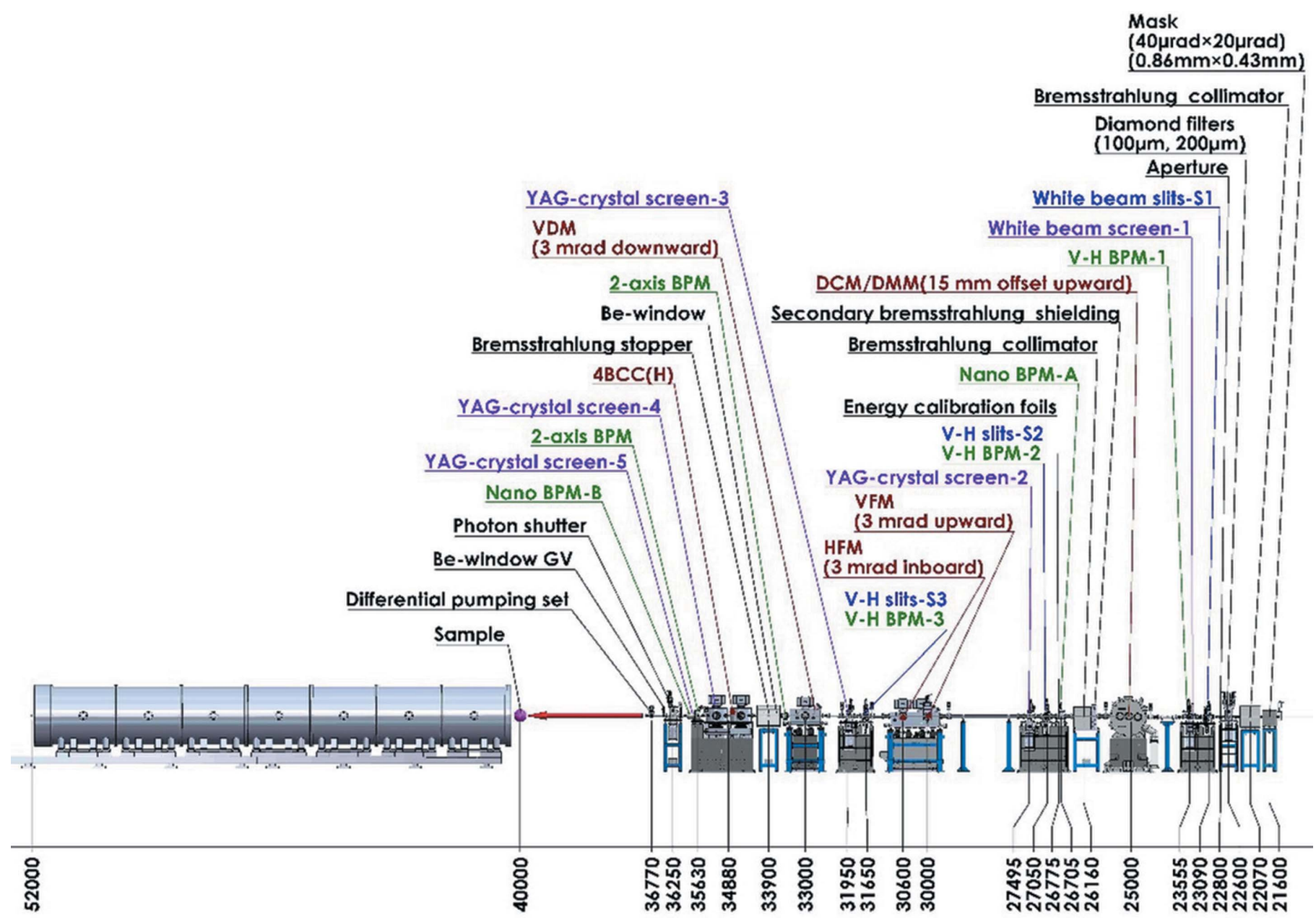

(a)

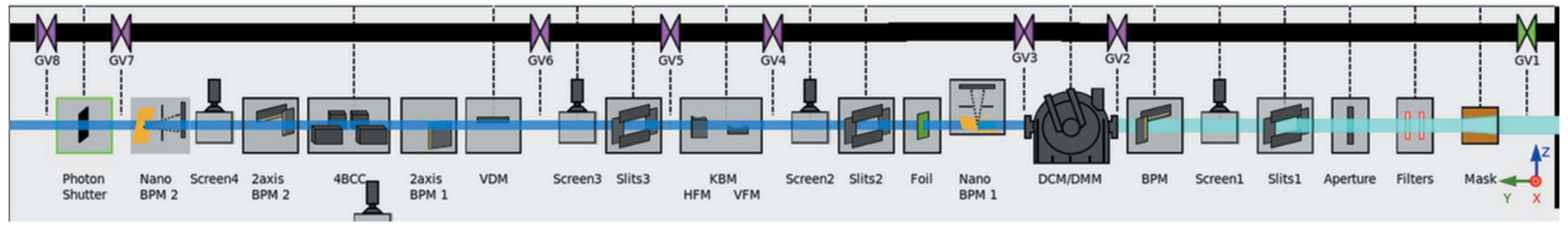

Figure 8

(b)

(a) Layout of the optical zone ( $21 \mathrm{~m}$ to $37 \mathrm{~m})$ and the endstation $(37-55 \mathrm{~m})$ of the TPS 13A beamline. (b) The CSS view of the optical zone. The relevant control dialog box would appear upon double-clicking the corresponding icon. VDM, vertical deflection mirror; BPM, beam-position monitor.

interface with controls and status displays of the beamline components. Fig. 8 shows the major components in the optical zone and the corresponding CSS interface for the components status and visualization controls.

SPEC macro scripts are successfully integrated into the EPICS to perform packaged and interactive operations, including sequential motions of motors, data collection and output for further processing, and input acquirements from different devices via their PVs embedded in the EPICS. For example, the MOVE-E macro could change and optimize the gap of IU24 for a specific X-ray energy, followed by adjusting and optimizing the DCM setting. The macro continues on refining the positions and beam incident angles of VFM and HFM to obtain optimized beam intensity and predefined beam position at the sample position. The powerful automation procedure is executed on the basis of interactive communications with ongoing feedbacks of the beam position and intensity reported by the two endstation XBPMs (Rigi and Civi-1 in Fig. 9 near the sample position. SPEC macro scripts are also applied to the automation of the DMM/DCM mode conversion and the auto-positioning of the beamline slits.

\subsection{Endstation}

The experimental endstation is separated from the optical zone by a lead-embedded wall at $36.5 \mathrm{~m}$. A differential pumping section ( $720 \mathrm{~mm}$ long with $10.7 \mathrm{~mm}$ diameter) bridges the ultrahigh vacuum $\left(<10^{-7}\right.$ torr $)$ of the $4 \mathrm{BCC}$ to the 


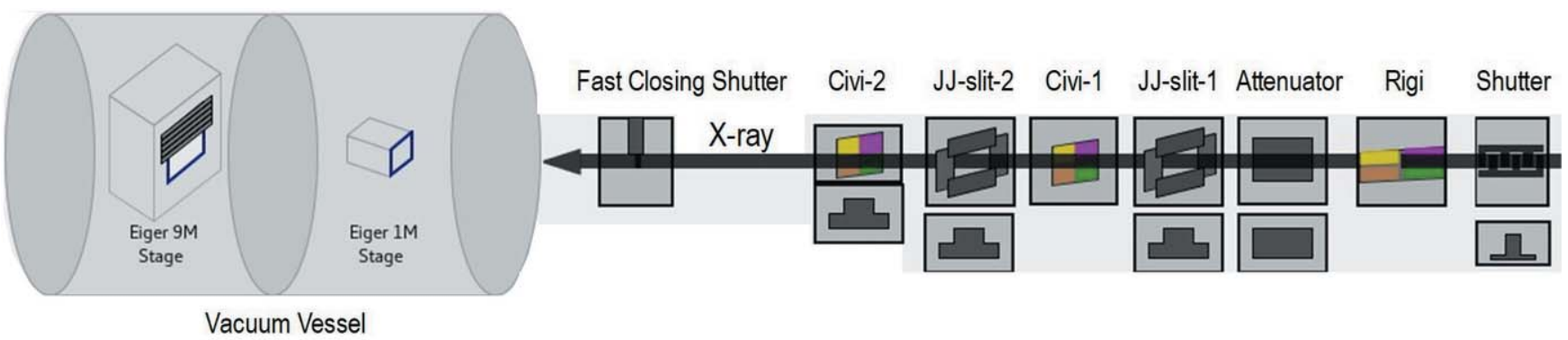

Figure 9

A CSS graphic view (not to scale) of the endstation zone, comprising (from the right) the primary components, including the three XBPMs of Rigi, Civi-1 and Civi-2. The left-hand-side is the detecting system with the two Eiger detectors X $1 \mathrm{M}$ and X 9M moving in a large vacuum vessel, $12 \mathrm{~m}$ in length and $1.5 \mathrm{~m}$ in diameter, for $\sim 10 \mathrm{~m}$ traveling distance along the beam path at a speed of $0.5 \mathrm{~m} \mathrm{~min}^{-1}$. The shortest camera distances are $710 \mathrm{~mm}$ and $180 \mathrm{~mm}$ for the two detectors, respectively. The Eiger X 9M could move $\pm 120 \mathrm{~mm}$ in the lateral and vertical directions, respectively, and is equipped with a $4 \mathrm{~mm}$ beamstop.

first vacuum section in the experimental hutch of higher pressure near $10^{-5}-10^{-6}$ torr. This differential pumping section provides a windowless path for the low-energy beam (mainly 4-5 keV X-rays) to waive beam intensity loss from absorption of a window. Nevertheless, for safety concerns, a UHV gate valve with $250 \mu \mathrm{m}$ Be window is installed downstream of the 4BCC to selectively isolate the beamline vacuum section from the experiment vacuum section for higher-energy $\mathrm{X}$-rays (of high Be-window transmission).

The first component of the experimental hutch is a vacuumtype fast shutter ( $8 \mathrm{~ms}$ shutter speed), which regulates the exposure of samples to the X-ray beam. Subsequently, an $\mathrm{X}$-ray intensity attenuator, modified from a gas-driven (6 bar) ADC ABS-300, a precision attenuator for hard X-rays (Oxford Co.), can selectively suppress the X-ray beam in 4$23 \mathrm{keV}$ across eight orders of magnitude in intensity. The attenuator could suppress the strong direct beam (up to $\sim 10^{14}$ photons $^{-1}$ ) for a direct beam image taken with the Eiger $X$ 9M, having a maximum pixel-counting rate of $3 \times 10^{6}$ photons $\mathrm{s}^{-1}$. The wide range of attenuation is achieved via ten specifically designed foils made of different thicknesses of $\mathrm{Cu}$, Ta and Al. A user-friendly CSS interface is developed to execute the comparison of an input (target) attenuation factor with all possible attenuation factors calculated from every combination of the ten foils; the calculations are completed through a Python program integrated into EPICS for accessing and sharing information on beam energy and foil transmissions/positions. After confirmation through the CSS interactive dialog box, the selected foils of a best matched attenuation factor are automatically sent into the beam path ( $c f$. Fig. 9). A silicon wafer $(10 \mathrm{~mm}$ by $10 \mathrm{~mm})$, deposited with a $100 \mathrm{~nm}$-thick silver layer, is positioned downstream inside the attenuator vacuum chamber. The wafer can be selectively moved into the beam path to reflect a laser beam onto the X-ray beam path defined by two sets of JJ $\mathrm{X}$-ray vacuum slits (JJ-slits). Namely, the laser beam path would be tuned to pass through the same two sets of JJ-slits, leading to overlapped X-ray and laser beam paths. This arrangement allows visual guiding on sample positioning for SAXS-WAXS measurements.

The first set of JJ-slits (tungsten carbide, WC, blades with edges glued with Ge crystals) situated at $39.2 \mathrm{~m}$ before the sample position and the set of all-tungsten slits (S3) positioned at $31.65 \mathrm{~m}$ in the optical zone are used as a major collimation of the X-ray beam. The second set of JJ-slits, of $200 \mathrm{~mm}$ mobility along the beam path, can dynamically approach the sample position to minimize the residual air path (source of background scattering) of the beam before entering the detecting chamber. On the two vertical blades of each JJ-slit, four Ta pinholes (of diameters between 0.15 and $0.4 \mathrm{~mm}$ ) are embedded for the option of pinhole-collimation (versus JJ-slit edge collimation). The pinhole positions to the beam are relatively easily allocated after the slits centering to the beam via automated centering SPEC macros.

The beam positions and intensities are monitored online with two four-quadrant-diamond XPBMs: (1) Rigi (quadrantdiamond sensor of $20 \mu \mathrm{m}$ gap from Dectris) and (2) Civi-1 ( $3 \mu \mathrm{m}$ gap, Cividec Co.), which are situated before and after the attenuator, respectively. Another set of XBPMs, Civi-2, was positioned at the sample position for beam size measurements only. These quadrant diamond XPBMs report beam intensity and position with $1 \mu \mathrm{m}$ resolution, via in-house electric current-reading systems with voltage-to-frequency converters; these readout data are parameterized into PVs of the EPICS, and are shared with CSS for online display and for beam intensity normalization in SAXS-WAXS data processing. Uniquely designed is an integrated detecting system comprising an Eiger X 9M detector for SAXS and an Eiger X $1 \mathrm{M}$ detector for WAXS. These two X-ray detectors, of the same pixel size, move independently with multiple degrees of freedom inside a large vacuum vessel of length $12 \mathrm{~m}$ and diameter $1.5 \mathrm{~m}$, providing dynamic and quick changes in the detecting configuration. The operation of all components in the endstation zone is integrated into the CSS interface, as shown in Fig. 9.

\subsection{Performance tests}

We tested the USAXS performance using ensemble gold arrays of 2000, 1000, 500 and $200 \mathrm{~nm}$ spacing (thickness $\sim 650 \mathrm{~nm})$ deposited on a silicon nitride $\left(\mathrm{Si}_{3} \mathrm{~N}_{4}\right)$ membrane (Applied Nanotools Inc.), as shown in the inset of Fig. 10(a). With a $6 \mathrm{keV}$ beam of DCM and 4BCC, a sample-to-detector distance of $9.47 \mathrm{~m}$, and a beamstop of $4 \mathrm{~mm}$ diameter, the 

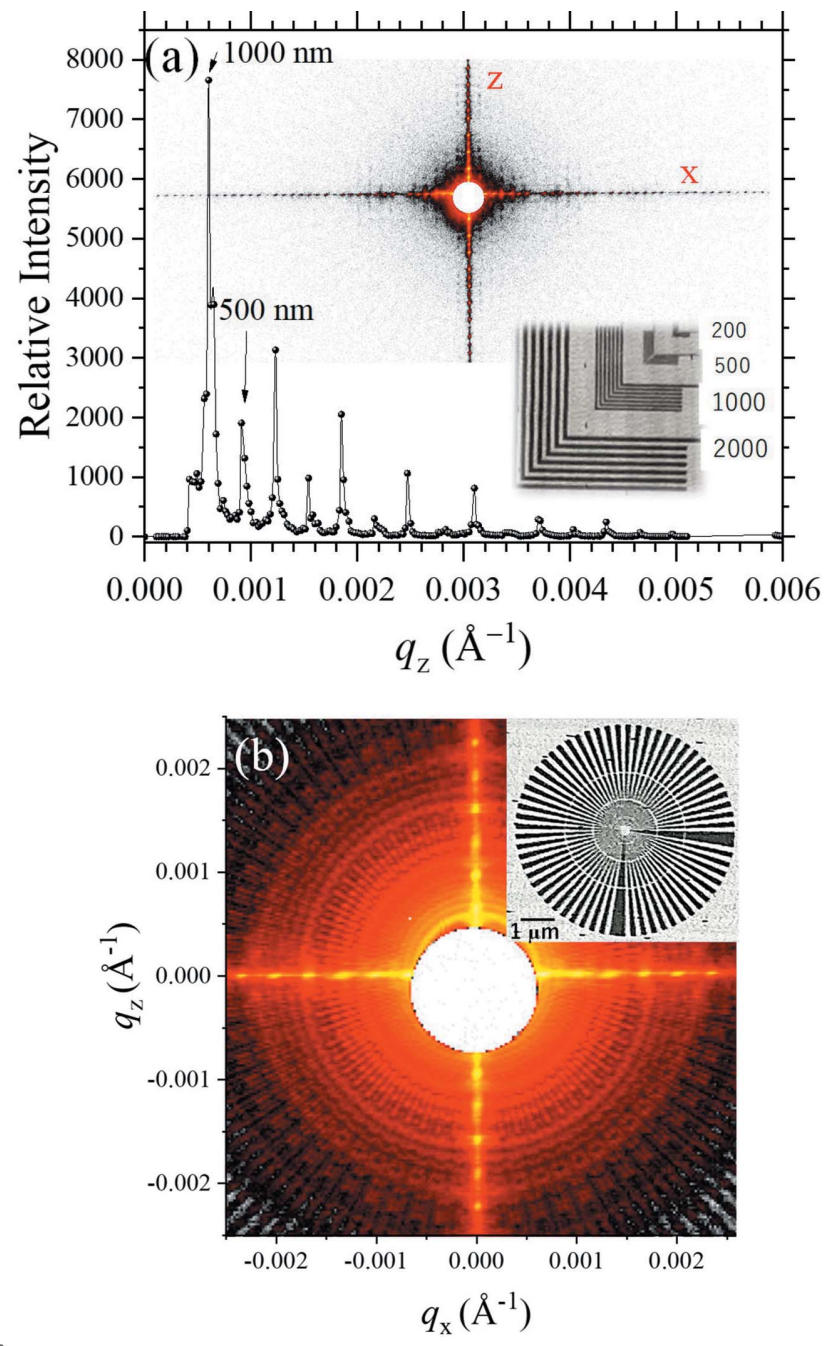

Figure 10

(a) USAXS pattern (inset) of an ensemble of gold L-shaped arrays of 2000, 1000, 500 and $200 \mathrm{~nm} d$-spacing (inset), measured using a $6 \mathrm{keV}$ beam of DCM with 4BCC. The line-cut profile taken along the $z$-axis reveals the primary peak of the array of $1000 \mathrm{~nm}$ spacing located at $q=$ $6.3 \times 10^{-4} \AA^{-1}$. The minimum detectable $q$ is $\sim 4.0 \times 10^{-4} \AA^{-1}$. (b) $\mathrm{A}$ USAXS pattern of a Siemens star pattern (inset), having $25 \mathrm{~nm}$ lines and spaces at the center zone. Note that the fine scattering features are differentiable down to $q \simeq 0.6 \times 10^{-4} \AA^{-1}$.

lowest detectable $q$ with the Eiger $\mathrm{X} 9 \mathrm{M}$ detector is $4.0 \times$ $10^{-4} \AA^{-1}$, which could resolve clearly the primary peak $q=$ $6.28 \times 10^{-4} \AA^{-1}$ of the $1000 \mathrm{~nm}$ spacing of the gold arrays. Here, $q$ is defined by $4 \pi \lambda^{-1} \sin \theta$ with $\mathrm{X}$-ray wavelength $\lambda$ and scattering angle $2 \theta$. Moreover, fine scattering details of a Siemens star pattern fabricated on the same $\mathrm{Si}_{3} \mathrm{~N}_{4}$ substrate, of $25 \mathrm{~nm}$ lines and spaces at the center zone, could be observed clearly as illustrated in Fig. $10(b)$, with a discernible $q$-resolution down to $0.6 \times 10^{-4} \AA^{-1}$ (matching the design $q$-resolution).

Fig. 11(a) demonstrates that the large Eiger X 9M (active area of $233 \mathrm{~mm} \times 245 \mathrm{~mm}$ ) could cover a wide $q$-range in one imaging for both SAXS and WAXS information, using a sample-to-detector distance of $710 \mathrm{~mm}$ and $10 \mathrm{keV}$ beam. With the detecting configuration, a highly asymmetric X-ray scattering pattern was taken from a turkey tendon. In the
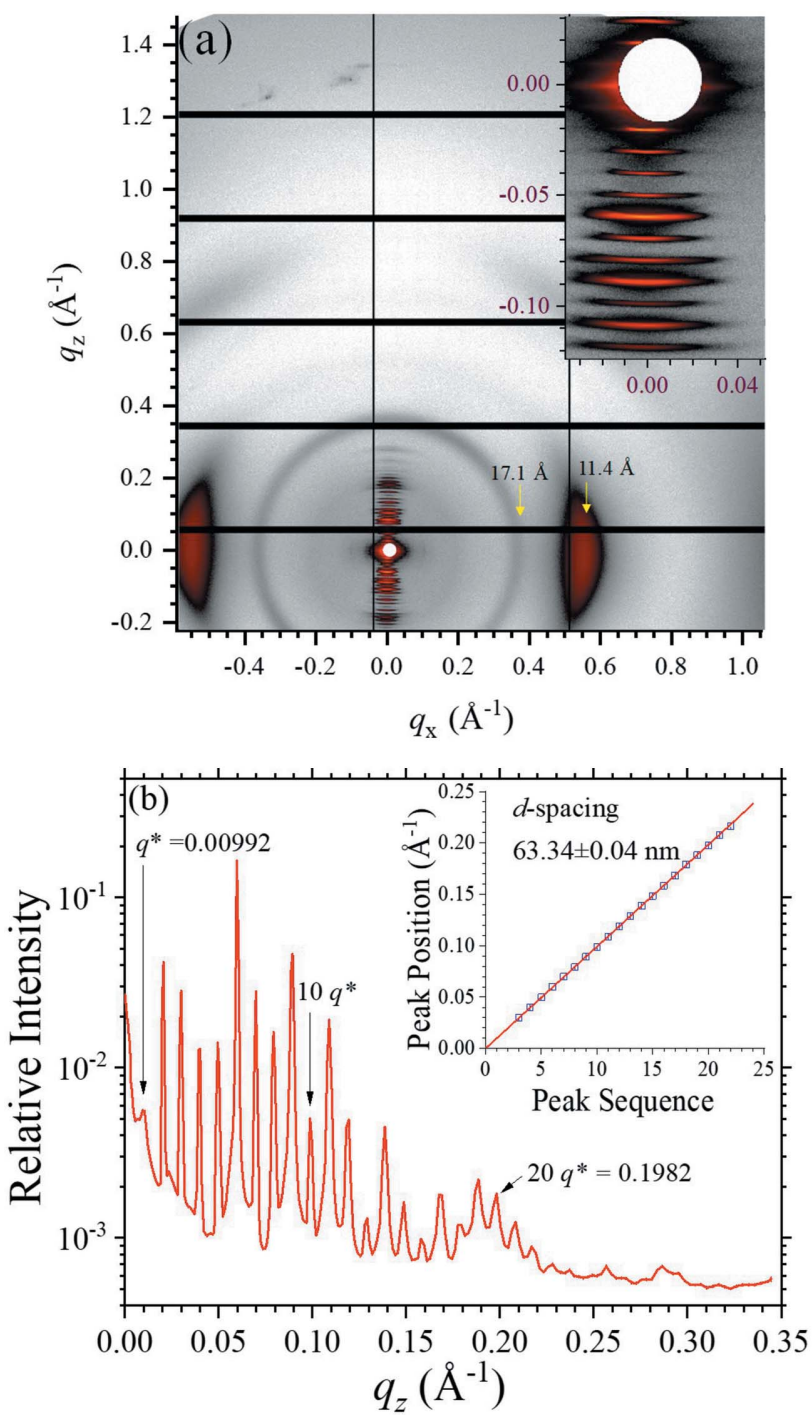

Figure 11

(a) Highly oriented 2D pattern taken from a turkey tendon, covering a wide $q$-range as indicated. The inset enlarges the details of the SAXS region for the periodic peaks along the $q_{z}$ direction. Two prominent arcs located along the $q_{x}$ axis are selectively marked with the corresponding lateral $d_{\mathrm{L}}$-spacing of $11.4 \AA$. (b) The intensity line-cut profile extracted along the $q_{z}$ axis, with the periodic peaks selectively numbered. The inset shows that all the peak positions fall nearly perfectly on the linear regression line, for the $d$-spacing indicated.

SAXS region, highly oriented periodic peaks are observed along the $q_{z}$ axis [Fig. 11(a)], revealing amazingly ordered packing of tropocollagen along the collagen fibril orientation (Maurya et al., 2021). Deduced from a linear fitting of the observed peak positions [inset of Fig. 11(b)], the first peak position is determined to be $0.00992 \AA^{-1}$ [Fig. 11(b)], corresponding to a $d$-spacing of $63.34 \pm 0.04 \mathrm{~nm}$. The corresponding ordered domains size estimated from the peak width is $\sim 4.0 \mu \mathrm{m}$. Moreover, perpendicularly oriented arcs (with respect to the fibril orientation) are observed at $q_{x}=$ $0.5526 \AA^{-1}$, corresponding to a characteristic Bragg $d$-spacing of $d_{\mathrm{L}}=11.4 \AA$. Assuming a 2D-hexagonal packing of the tropocollagen in the cross-sectional direction, we could deduce a lattice constant $a=d_{\mathrm{L}} /(\sqrt{3} / 2)$ of $13.3 \AA$, which 
corresponds to the center-to-center distance of the 2D-hexagonally packed tropocollagen of the turkey tendon (Fang et al., 2012). Our result demonstrates that orientations and ordered sizes of hierarchically ordered structures could be correlated conveniently and faithfully in one single imaging.

\section{Conclusion}

The TPS 13A biological SAXS beamline has demonstrated its prominent facilities including high flux, USAXS and microbeam. The high flux above $10^{14}$ photons s ${ }^{-1}$ for studies of structures and kinetics is enabled with the $4 \mathrm{~m}$-long IU24 source and an efficient double-multilayer monochromator; the USAXS is enabled with a long camera length of $10 \mathrm{~m}$ and a low X-ray energy beam with small beam divergence and low scattering background, achieved with the combined vertically oriented DCM and horizontally oriented $4 \mathrm{BCC}$; these together allow resolving hierarchically ordered structures up to $1 \mu \mathrm{m}$ length scale. A microbeam of $10 \mu \mathrm{m}$ beam size and small beam divergence for structural mappings with simultaneous SAXS/WAXS measurements is achieved with microslits situated in the beamline front-end and a set of dedicated KB mirrors. All these salient features are integrated into the beamline via coordinated optical systems of similar beam paths, which is critical for the multi-mode beamline operations. Graphic CSS and SPEC macro scripts are successfully integrated into the EPICS system for complementary and streamline beamline controls, ranging from change of X-ray energy with IU24 to data collection with the two Eiger detectors.

\section{Acknowledgements}

The authors thank Drs N. Yagi, H. Masunaga, J. Perez, T. Narayanan, C. Blanchet and D. Svergun for valuable suggestions. The help from C. M. Cheng and C.-C. Chang on the front-end vacuum, $\mathrm{XBPM}$ and $\mathrm{LN}_{2}$ cooling system is acknowledged. Substantial beamline construction support from Drs K.-L. Yu and Y.-S. Huang are acknowledged.

\section{Funding information}

Funding from the Academia Sinica and the Taiwan Ministry of Science and Technology through a flagship project is acknowledged.

\section{References}

Blanchet, C. E., Spilotros, A., Schwemmer, F., Graewert, M. A., Kikhney, A., Jeffries, C. M., Franke, D., Mark, D., Zengerle, R.,
Cipriani, F., Fiedler, S., Roessle, M. \& Svergun, D. I. (2015). J. Appl. Cryst. 48, 431-443.

Chiang, L. C., Chen, H. W., Liao, B. Y., Liu, C. Y. \& Chang, C. F. (2019). AIP Conf. Proc. 2054, 060056.

Chuang, J. Y., Lin, Y. Z., Hsiao, Y. M., Liu, Y. C., Shu, D., Kuan, C. K., Wu, H., Sheng, I. C., Cheng, Y. T., Shueh, C., Chang, C. C., Chan, C. K. \& Yen, J. Y. (2020). Nucl. Instrum. Methods Phys. Res. A, 953, 163174-163175.

Cowieson, N. P., Edwards-Gayle, C. J. C., Inoue, K., Khunti, N. S., Doutch, J., Williams, E., Daniels, S., Preece, G., Krumpa, N. A., Sutter, J. P., Tully, M. D., Terrill, N. J. \& Rambo, R. P. (2020). J. Synchrotron Rad. 27, 1438-1446.

Desjardins, K., Bordessoule, M. \& Pomorski, M. (2018). J. Synchrotron Rad. 25, 399-406.

Fang, M., Goldstein, E. L., Turner, A. S., Les, C. M., Orr, B. G., Fisher, G. J., Welch, B. K., Rothman, E. D. \& Banaszak Holl, M. M. (2012). ACS Nano, 6, 9503-9514.

Hatje, J., Clausen, M., Gerke, C., Moeller, M. \& Rickens, H. (2007). Proceedings of the 11th International Conference on Accelerator and Large Experimental Physics Control Systems (ICALEPCS2007), 15-19 October 2007, Knoxville, TN, USA, pp. 37-39.

Horiuchi, N. (2015). Nat. Photon. 9, 292-293.

Hsu, C. H., Jeng, U., Lee, H. Y., Huang, C. M., Liang, K. S., Windover, D., Lu, T. M. \& Jin, C. (2005). Thin Solid Films, 472, 323-327.

Jeng, U.-S., Su, C. H., Su, C.-J., Liao, K.-F., Chuang, W.-T., Lai, Y.-H., Chang, J.-W., Chen, Y.-J., Huang, Y.-S., Lee, M.-T., Yu, K.-L., Lin, J.-M., Liu, D.-G., Chang, C.-F., Liu, C.-Y., Chang, C.-H. \& Liang, K. S. (2010). J. Appl. Cryst. 43, 110-121.

Lai, Y.-H., Sun, Y. S., Jeng, U., Huang, Y. S., Song, Y. F., Dronyak, R., Tsang, K. L. \& Liang, K. S. (2005). Nucl. Instrum. Methods Phys. Res. B, 238, 205-213.

Lai, Y.-H., Sun, Y.-S., Jeng, U.-S., Lin, J.-M., Lin, T.-L., Sheu, H.-S., Chuang, W.-T., Huang, Y.-S., Hsu, C.-H., Lee, M.-T., Lee, H.-Y., Liang, K. S., Gabriel, A. \& Koch, M. H. J. (2006). J. Appl. Cryst. 39, 871-877.

Li, N., Li, X., Wang, Y., Liu, G., Zhou, P., Wu, H., Hong, C., Bian, F. \& Zhang, R. (2016). J. Appl. Cryst. 49, 1428-1432.

Liu, D.-G., Chang, C.-H., Lee, M.-H., Liu, C.-Y., Chang, C.-F., Chiang, L.-C., Hwang, C.-S., Huang, J.-C., Sheng, A., Kuan, C.-K., Yeh, Y.-Q., Su, C.-J., Liao, K.-F., Wu, W.-R., Shih, O. \& Jeng, U. (2019). AIP Conf. Proc. 2054, 060021.

Liu, D.-G., Chang, C.-H., Liu, C.-Y., Chang, S.-H., Juang, J.-M., Song, Y.-F., Yu, K.-L., Liao, K.-F., Hwang, C.-S., Fung, H.-S., Tseng, P.-C., Huang, C.-Y., Huang, L.-J., Chung, S.-C., Tang, M.-T., Tsang, K.-L., Huang, Y.-S., Kuan, C.-K., Liu, Y.-C., Liang, K. S. \& Jeng, U.-S. (2009). J. Synchrotron Rad. 16, 97-104.

Maurya, A. K., Parrilli, A., Kochetkova, T., Schwiedrzik, J., Dommann, A. \& Neels, A. (2021). Acta Biomater. 129, 169-177.

Petoukhov, M. V., Franke, D., Shkumatov, A. V., Tria, G., Kikhney, A. G., Gajda, M., Gorba, C., Mertens, H. D. T., Konarev, P. V. \& Svergun, D. I. (2012). J. Appl. Cryst. 45, 342-350.

Sanchez del Rio, M., Canestrari, N., Jiang, F. \& Cerrina, F. (2011). J. Synchrotron Rad. 18, 708-716.

Sztucki, M., Leonardon, J., Van Vaerenbergh, P., Gorini, J., Boesecke, P. \& Narayanan, T. (2019). J. Synchrotron Rad. 26, 439-444.

Tanaka, T. \& Kitamura, H. (2001). J. Synchrotron Rad. 8, 1221-1228. 